\title{
Multilingual Approach to COVID-19 Online Learning Response on OpenWHO.org
}

\author{
Heini UTUNEN ${ }^{\mathrm{a}, 1}$, Ngouille NDIAYE ${ }^{\mathrm{a}}$, Melissa ATTIAS ${ }^{\mathrm{a}}$, Lama MATTAR ${ }^{\mathrm{a}}$, \\ Anna TOKAR ${ }^{\mathrm{a}}$ and Gaya GAMHEWAGE ${ }^{\mathrm{a}}$ \\ ${ }^{\text {a }}$ World Health Organization, Geneva, Switzerland
}

\begin{abstract}
In pursuit of equitable access to emergency-related knowledge, the World Health Organization (WHO) translates COVID-19 and other infectious disease courses into multiple languages on its open-access online learning platform OpenWHO.org. Languages spoken by vulnerable or underserved populations in low- and middle-income countries and in outbreak-prone and affected areas are prioritized. Accessing learning in preferred languages enhances uptake and comprehension. In this study, we assess and compare the initial enrollment levels and global reach of these multilingual courses. On average, OpenWHO's 38 COVID- 19 courses have each been translated into 4.8 languages. The platform hosts courses in 55 different languages with 10.4 million words translated. The findings identify which available languages were most utilized for COVID-19 learning to inform course production and outreach strategies. Languages were used differently across geographic regions, calling for localized learning offerings. A streamlined multilingual publishing scheme, ensuring quick and effective delivery of diverse languages, is critical to achieving greater equity of access to knowledge.
\end{abstract}

Keywords. OpenWHO, multilingual, online learning, COVID-19 pandemic

\section{Introduction}

OpenWHO.org is an open-access, low-bandwidth online learning platform created by the World Health Organization's Health Emergencies Programme (WHE) in 2017 to democratize global access to trusted public health knowledge. The platform is designed to support preparedness and response to outbreaks [1]. OpenWHO has delivered critical learning content during the pandemic and ensured broader equity through courses in 55 languages, including the official languages of every WHO region, the 15 most-spoken languages worldwide, 14 African languages and the official languages of 42 out of 46 of the least-developed countries.

WHE has been producing materials for emergencies since the West Africa Ebola outbreak. The solutions and services put into practice include local language translation capacity, unlimited online dissemination to the front lines of emergencies through a dedicated low-bandwidth platform, and a process to turn evidence-based, emergency guidelines into knowledge resources for responders. The World Health Assembly (2019) was convinced of the importance of respecting the diversity of cultures and the plurality

\footnotetext{
${ }^{1}$ Corresponding Author, Heini Utunen; E-mail: utunenh@who.int.
} 
of international languages. This was echoed in the United Nations Joint Inspection Unit report on multilingualism (2020) [2].

Comprehension can be greatly improved when using the appropriate languages and formats [3]. Learning in one's preferred language enhances learning effectiveness [4]. This is particularly true for fast-changing epidemic and outbreak contexts, as well as in humanitarian settings with multiple health concerns for affected populations [5].

This paper presents the multilingual approach pioneered by WHO's OpenWHO.org learning platform for health emergencies and the COVID-19 pandemic. It examines which languages are most utilized for COVID-19 learning and language use patterns by geographic region to inform and strengthen the platform's approach.

\section{Methods}

Data were extracted from the built-in OpenWHO.org reporting system and a descriptive analysis was conducted to assess early course traction by language and geographic spread from 26 January 2020 to 26 August 2021. OpenWHO user metrics included self-reported data on location, gender, language, age and affiliation of users, which may be utilized for research under the platform terms of use. As this study was deemed 'non-invasive', ethical clearance was not required.

Platform and course-based data were integrated to depict global and local use of COVID-19 courses by language. Course registrations were segregated by language used to access the course and by geographic location mapped according to WHO region. The first four weeks of course availability were targeted to establish a comparable baseline, as course topics and language versions are published at different times. To account for variations in language availability per course topic, each language's total enrollment count was divided by the number of topics available in that language.

\section{Results}

Multilingualism in OpenWHO's large-scale online production strategy has resulted in more than 5.6 million course enrollments driven largely by 38 COVID-19 courses, with 2.9 million certificates awarded for course completion or achievement. Courses are available in 55 languages, with a total of 10.4 million words translated thus far, hosted across 113 course topics. On average, OpenWHO's COVID-19 courses have each been translated into 4.8 languages, with the Introduction to COVID-19 course available in 44 languages and the Infection Prevention and Control course in 24 languages.

The top languages by enrollment are English (76.2\%), Spanish (13.0\%), French (3.1\%), Arabic (1.5\%), Portuguese (1.5\%), Indian sign language (1.0\%), Hindi $(0.7 \%)$, Indonesian $(0.6 \%)$, Russian $(0.5 \%)$ and Italian $(0.4 \%)$. All other languages combined constitute $1.5 \%$ of use.

English language course use varies significantly by WHO region. The WHO Region of the Americas is characterized by Spanish (48.0\%), English (46.7\%) and Portuguese $(2.8 \%)$ use. Enrollments in the English learning resources for other WHO regions are as follows: European (69.2\%), Eastern Mediterranean (84.1\%), South-East Asia (88.2\%), African (90.6\%) and Western Pacific (94.6\%). Spanish is the second most popular language among OpenWHO learners, with $14.5 \%$ of all enrollments in Spanish-language courses. 
Average enrollments per course by language in the first four weeks following course launch are shown in Table 1.

Table 1. Top languages by first four-week average enrollments per COVID-19 course.

\begin{tabular}{cccc}
\hline Languages & Enrollments & Languages & Enrollments \\
\hline English & 26327 & Chinese & 783 \\
Spanish & 12628 & Bengali & 661 \\
French & 7510 & Turkish & 503 \\
Portuguese & 3878 & Serbian & 417 \\
Arabic & 2052 & Japanese & 380 \\
Indian Sign & 1877 & Persian & 359 \\
Hindi & 1862 & Urdu & 352 \\
Indonesian & 1667 & Hungarian & 328 \\
Russian & 1151 & Oriya & 277 \\
Italian & 878 & German & 269 \\
\hline
\end{tabular}

\section{Discussion}

The OpenWHO platform ramped up learning production for the pandemic, deploying record-setting crowdsourcing power in its efforts to deliver life-saving knowledge with an emphasis on multilingualism to cater to the demands of a massive global audience. Examining the initial enrollment surge by language in the first four weeks after course launch helps account for the distribution of courses across languages - courses are published more often in some languages than others - and variations in course publication date, as courses published earlier have more overall enrollments. It also highlights the traction of languages such as Indian sign language with notable levels of popularity. The importance of national and local language resources far exceeds the use percentage in terms of providing equitable access to learning for the most vulnerable and contributing to co-ownership.

The results inform OpenWHO's multilingual approach by suggesting which languages are in highest demand for COVID-19 learning for future course production, as well as which languages should be targeted for outreach and promotion to reach additional learners. Geographically, the study shows that English courses are used in a much lower proportion in the Americas and Europe and at a much higher rate in Africa and the Western Pacific This suggests that research into the drivers of these regional variations, whether unmet language needs or limited platform reach, could further strengthen the multilingual production strategy. Overall, the importance of Spanish among learners has implications for learning production and confirms the urgency of timely Spanish translation. The popularity of Indian sign language, the seventh most popular language on the platform, illustrates the demand for learning resources by special needs audiences.

The scalability of OpenWHO's response was achieved through a fast-paced production system with strengthened commitment to equity of access. This was bolstered through local and co-ownership, as WHO country offices, health institutes, individuals and other volunteers translated the materials to meet their needs and further adapted them for field use in plain language and other localized formats. A multiplier effect has occurred with national language materials in particular, as the translated and adapted learning resources have been used extensively outside of the platform context. 
One limitation of the data is that the English version of a course is launched first, likely contributing to the higher uptake in the first four weeks as it may be the only language version available. This might have led to an unequal distribution of enrollments across languages. Nevertheless, the findings testify to the importance of multilingualism and inform the translation planning process to support global preparedness and response with wider equity in access to trusted public health knowledge.

\section{Conclusion}

WHO's health emergency online learning platform supports global COVID-19 preparedness and response while seeking enhanced health literacy through multilingualism. The translation production system has successfully scaled up to meet the global demand for learning during the pandemic and can be further refined based on usage patterns. Additional work is required to study comprehension and assess communication preferences across affected and at-risk populations. This area continues to evolve to support more efficient and equitable access to a life-saving knowledge platform.

\section{References}

[1] Utunen H, Attias M, George R, Ndiaye N, Piroux C, Farzi MR, Gamhewage G. Global Access to OpenWHO's Online Learning Resources for COVID-19. Stud Health Technol Inform. 2020 Jun;272:304305.DOI:10.3233/shti200555.

[2] Lozinskiy N. Report of the Joint Inspection Unit. Multilingualism in the United Nations system. United Nations. 2020 Jun; Geneva, Switzerland: The United Nation.

[3] Nwokediuko AA. Advancing e-learning in African native communities: The language factor. International Conference on Education and e-Learning Innovations. 2012 Jul;1-4. DOI: 10.1109/ICEELI.2012.6360594.

[4] Watkins PG, Razee H, Richters J. (2012). 'I'm Telling You ... The Language Barrier is the Most, the Biggest Challenge': Barriers to Education among Karen Refugee Women in Australia'. Australian Journal of Education. 2012 Aug;56(2).

[5] Finset A, Bosworth H, Butow P, Gulbrandsen P, Hulsman RL, Pieterse AH, Street R. Tschoetschel R, van Weert J. Effective health communication - a key factor in fighting the COVID-19 pandemic. Patient Education and Counseling. 2020 May;103(5):873-876. 